\title{
Editorial : Hepatic Cirrhosis Clinical and Pathological Association; What is beyond? \\ Talaat Fathy
}

Tropical Medicine Department, Faculty of Medicine, Zagazig University, Zagazig, Egypt

talaat_Fathy1972@yahoo.com

\section{See the article pages: $249-256 ; 257-263$}

\section{Background}

Cirrhosis is characterized by fibrosis and nodule formation of the liver, secondary to a chronic injury, which leads to alteration of the normal lobular organization of the liver. Various insults can injure the liver, including viral infections, toxins, hereditary conditions, or autoimmune processes. With each injury, the liver forms scar tissue (fibrosis), initially without losing its function. After a longstanding injury, most of the liver tissue gets fibrosed, leading to loss of function and the development of cirrhosis [1].

Chronic liver diseases usually progress to cirrhosis. In the developed world, the most common causes of cirrhosis are hepatitis $\mathrm{C}$ virus (HCV), alcoholic liver disease, and nonalcoholic steatohepatitis (NASH) [1], while hepatitis B virus (HBV) and HCV are the most common causes in the developing world. Other causes of cirrhosis include autoimmune hepatitis, primary biliary cholangitis, primary sclerosing cholangitis, hemochromatosis, Wilson disease, alpha-1 antitrypsin deficiency, Budd-Chiari syndrome, drug-induced liver cirrhosis, and chronic right-sided heart failure. Cryptogenic cirrhosis is defined as cirrhosis of unclear etiology.

Patients with cirrhosis can be asymptomatic or symptomatic, depending on whether their cirrhosis is clinically compensated or decompensated. In compensated cirrhosis, patients are usually asymptomatic. On the other hand, patients with decompensated cirrhosis usually present with a wide range of signs and symptoms arising from a combination of liver dysfunction and portal hypertension. Multiple organs are affected with gastrointestinal, renal, pulmonary, cardiological and endocrine manifestations [1].

\section{Cardiac Changes in Cirrhotic Patients}

Liver cirrhosis is associated with a wide range of cardiovascular changes including hyperdynamic circulation, pulmonary vascular abnormalities, and cirrhotic cardiomyopathy. The pathogenesis of these cardiovascular changes is multifactorial and includes neurohumoral and vascular dysregulations [2].

According to an interesting study published in the current issue of the Afro-Egyptian Journal of Infectious and Endemic Diseases; "Cardiac Changes in Cirrhotic Patients", $70 \%$ of the studied patients with liver cirrhosis have cardiac dysfunction in the absence of other risk factors for cardiac diseases. In addition, the degree of cardiac dysfunction is correlated with the severity of liver disease of cirrhotic patients.

Hyperdynamic circulation is one of many cardiovascular abnormalities that occur in patients with liver cirrhosis [3]. Cirrhotic Cardiomyopathy describes the impairment of cardiac contraction in response to stress, diastolic dysfunction and electrophysiological changes in the absence of any cardiac disease in cirrhotic patients [4].

The blood pressure, according to that study, was significantly lower among Child B and Child $\mathrm{C}$ group cirrhotic patients compared to Child A cirrhotic patients. This comes in agreement with the result of Baik et al. [5]. Low blood pressure is probably attributed to the arterial vasodilatation that commonly found among cirrhotics [6]. 
QTC interval was found to be prolonged in $70 \%$ of cirrhotic patients. QTC interval was significantly prolonged with the progression of liver disease. This prolongation may be due to alterations of ion channel activity in cardiomyocyte plasma membranes [7].

Pulmonary artery pressure (PAP), in concern to that study, was increased in Child B and C patients when compared to Child A patients. This result agrees with that of Silvestre et al. [8] who found significant correlation between Child score and PAP. Increasing the levels of vasoactive substances circulating in the pulmonary circulation leads to pulmonary hypertension. These substances possibly induce vasoconstriction, with a possible toxic effect on endothelial cells [9].

That study revealed that there was no statistically significant difference regarding right ventricular end diastolic diameter among the studied groups. This result was going parallel to that of Soyoral et al. [10] who found that there was no change in right ventricular diameters, but it disagrees with that of Chen et al. [11] who demonstrated that the right ventricular dimension is significantly increased in patients with cirrhosis. This could be explained by an increased venous return to the right side of the heart caused by the development of portosystemic collaterals [12].

On the other hand, left ventricular end diastolic volume, left ventricular end systolic volume, LV end systolic diameter and LV end diastolic diameter increased in (Child B and C patients) when compared to (Child A patients) but not statistically significant. These abnormal structural findings in cirrhotic patients seem to be an adaptation of cardiac hemodynamics to changes in the peripheral circulation with diastole [13].

That study revealed that EF was decreased in Child B and C patients compared to Child A patients but not statistically significant and this agrees with the results found by Salari et al. [14]. This could be explained by that in advanced cirrhosis, there is an increase in blood volume that leads to a constant increase in cardiac output, which may cause cardiac overload, with impaired cardiac contractility and insufficient ventricular reserve response to an increase in ventricular filling pressure [15].

Diastolic dysfunction in cirrhotic patients has been linked with increased left ventricular wall thickness, subendocardial oedema, fibrosis and distorted collagen composition, ultimately leading to altered relaxation [16].

\section{Assessment of Thyroid Dysfunction in Patients with Chronic Hepatitis C Virus Related Liver Diseases}

HCV has extrahepatic features in multiple organ systems such as dermatologic, hematologic, renal, rheumatologic and endocrinal [17]. Of this most important endocrinal affection is thyroid abnormalities [18]. The liver has an essential role in the metabolism of thyroid hormones; peripheral conversion of tetraiodothyronine (T4) to triiodothyronine (T3) by type one deiodinase [19]. Previous studies claimed that levels of thyroid hormones, in addition to their binding proteins are disturbed among patients with hepatic disorders, especially cirrhotic. It is reported that the reduction in whole T3 and FT3 levels is the most frequent change in plasma levels of thyroid hormones, which is linked to the impact of hepatic derangement [20].

According to the study of 'Assessment of Thyroid Dysfunction in Patients with Chronic Hepatitis C Virus Related Liver Diseases'; published in the current issue of the AfroEgyptian Journal of Infectious and Endemic Diseases; cirrhotic hepatitis $\mathrm{C}$ patients have thyroid abnormalities higher than non-cirrhotic patients do. It is intensely related to the severity of liver affection and the advancing of Child score. Serum bilirubin, prothrombin time, duration of disease and degree of liver cirrhosis are dependent risk factor for TSH abnormalities. TSH, FreeT4 and Free T3 can be considered to be useful prognostic indices.

The result of that research revealed a decrease in the level of FT3 in cirrhotic patients and it 
was statistically significant. When the serum levels of FT3 in different subgroups were compared, Child A group was within the normal range meanwhile Child $\mathrm{C}$ patients had the lowest levels.

These results also revealed that the level of FT4 was statistically significant lower in cirrhotic patients. When comparing the mean serum level of FT4 they found that the lowest levels were in Child $\mathrm{C}$ group, followed by the Child B group, meanwhile Child A group was within the normal range.

They proposed that the previous findings might be reliant on the severity of hepatocellular damage and might be reversed on improvement of liver functions, so that study considered FT4 and FT3 levels as useful prognostic indices.

That study revealed a highly significant level of TSH in cirrhotic patients when compared to non-cirrhotic. When comparing the mean TSH serum level in different subgroups; they found that Child $\mathrm{C}$ group had the highest level followed by the Child B group, meanwhile normal range was found in Child A group. These different results might be due to differences in HCV genotyping, environmental factors, geographic distribution, and genetic background. That study showed that serum albumin level, serum bilirubin, prothrombin time and Child-Turcotte-Pugh score are dependent risk factor for FT3 and FT4 abnormalities. These results are similar to those of Borzio et al., [21] who observed that serum FT3 was significantly correlated with serum bilirubin, serum albumin, and Prothrombin time.

\section{References:}

1. Naveau S, Perlemuter G, Balian A. Epidemiology and natural history of cirrhosis. Rev Prat. 2005 30; 55(14):1527-32.

2. Raval Z, Harinstein ME, Skaro A, Erdogan A, De Wolf AM, Shah SJ, et al. Cardiovascular Risk assessment of the Liver Transplant Candidate. J Am Coll Cardiol 2011; 58(3): 223-31.
3. Moezi L, Mehr SE, Dehpour AR. Cardiovascular Abnormalities in Cirrhosis: The Possible Mechanisms. J Teh Univ Heart Ctr 2007; 4: 191-200.

4. Møller S, Henriksen JH. Cirrhotic cardiomyopathy. J Hepatol 2010; 53:179-190.

5. Baik SK, Fouad TR, Lee SS. Cirrhotic cardiomyopathy. Orphanet J. Rare Dis 2007; 27: 2-15.

6. Møller S, Henriksen JH. Cardiovascular complications of cirrhosis. Gut 2008; 57:268278.

7. Ward CA, Liu H, Lee SS. Altered cellular calcium regulatory systems in a rat model of cirrhotic cardiomyopathy. Gastroenterology 2001; 121: 1209-18.

8. Silvestre OM, Bacal F, De Souza Ramos D, Andrade JL, Furtado M, Pugliese V, et al. Impact of the severity of end-stage liver disease in cardiac structure and function. Ann Hepatol 2013; 12(1):85-91.

9. Khan A, Al-Jahdali H, Abdullah K, Irion K, Sabih Q, Gouda A. Pulmonary vascular complications of chronic liver disease: Pathophysiology, imaging, and treatment. Ann Thorac Med. 2011;6(2):57-65.

10. Soyoral Y, Süner A, Kıdır V, Arıtürk Z, Balakan O, Halil Değertekin H. The effect of viral cirrhosis on cardiac ventricular function. Eur J Gen Med 2004; 1(2): 15-18.

11. Chen Y, Chan AC, Chan S-C, Chok S-H, Sharr W, Fung J, et al. A detailed evaluation of cardiac function in cirrhotic patients and its alteration with or without liver transplantation. J Cardiol. 2016;67(2):140-146..

12. Zardi EM, Dobrina A, Ambrosino G, Margiotta D, Polistina F, Afeltra A. New therapeutic approaches to liver fibrosis: apracticable route? Curr Med Chem 2008; 15: 1628-44.

13. Alqahtani SA, Fouad TR, Lee SS. Cirrhotic cardiomyopathy. Semin Liver Dis 2008; 28(1):5- 69. 
14. Salari A, Shafaghi, Ofoghi M, Saeidinia A, Mansour-Ghanae F. Diastolic dysfunction and severity of cirrhosis in nonalcoholic cirrhotic patients. Int J Hepatol 2013; 1-6.

15. Møller S, Henriksen JH. Liver cirrhosis and arterial hypertension. World $J$ Gastroenterol 2006; 12(5).

16. Mahadevan G, Dwivedi G,Williams L, Steeds RP. FrenneauxM. Epidemiology and diagnosis of heart failure with preserved left ventricular ejection fraction: rationale and design of the study. Eur J Heart Fail 2012; 14:106-12.

17.Soliman AM , El hawari SA, Refaey MM, Ahmed NH, Emara MH. Extrahepatic Manifestations of Hepatitis C Virus: An Extending List. Afro-Egypt J Infect Endem Dis 2012; 2(1): 36-53.
18. Jadali Z. Autoimmune thyroid disorders in hepatitis $C$ virus infection: Effect of interferon therapy. Indian Journal of Endocrinology and Metabolism 2013; 17(1): 69.

19. Kharb S, Garg MK, Puri P, Sharad S. Assessment of Thyroid and Gonadal Function in Liver Diseases. Indian Journal of Endocrinology and Metabolism 2015; 19: 8994.

20. Lin ZH, Xin YN, Dong QJ, Wang Q, Jiang $\mathrm{XJ}$, Zhan $\mathrm{SH}$, et al. Performance of the aspartate aminotransferase-to-platelet ratio index for the staging of hepatitis C-related fibrosis: an updated meta-analysis. Hepatology 2011; 53: 726-36.

21. Borzio M, Caldara R, Borzio F, Piepoli V, Rampini $\mathrm{P}$, and Ferrari $\mathrm{C}$. Thyroid function tests in chronic liver disease: evidence for multiple abnormalities despite clinical euthyroidism. Gut 1983; 24(7): 631-636. 